Sitzungsber. Abt. II (2007) 216: 127-134 Sitzungsberichte

Mathematisch-naturwissenschaftliche Klasse Abt. II

Mathematische, Physikalische und Technische Wissenschaften

C Österreichische Akademie der Wissenschaften 2008

Printed in Austria

\title{
Zur Kombinationswirkung von Schall und Erschütterungen
}

\author{
Von \\ Peter Steinhauser \\ (Vorgelegt in der Sitzung der math.-nat. Klasse am 13. Dezember 2007 \\ durch das k. M. I. Peter Steinhauser)
}

\begin{abstract}
Zusammenfassung
Den aktuellen Diskussionen im Bereich des Lärmschutzes folgend wird der Stand der Forschung zur Kombinationswirkung von Lärm und Erschütterungen auf den Menschen zusammenfassend dargestellt. Insbesondere die unterschiedlichen Wahrnehmungsformen - Erschütterungen über den Körper, Schall durch das Ohr erschweren experimentelle Untersuchungen. Gesicherte Ergebnisse sind, dass der Mensch bei energetisch schwachen Immissionen (Verkehr) zwischen Hören und Spüren nicht unterscheiden kann. Eine Kombinationswirkung ist erst ab $W_{m}$ bewerteten Schwingbeschleunigungen von $30 \mathrm{~mm} / \mathrm{s}^{2}$ und A-bewerteten Schallpegeln von $45 \mathrm{~dB}$ nachgewiesen. Ab etwa $100 \mathrm{~mm} / \mathrm{s}^{2}$ bzw. $65 \mathrm{~dB}$ werden Verdeckungseffekte für die jeweils andere Wahrnehmungsform wirksam. Untersuchungen zur Quantifizierung der Kombinationswirkung im betroffenen Immissionsband stehen noch aus. Im Vergleich zur Immissionswirkung von Schall und Erschütterungen alleine dürfte die Kombinationswirkung aber nur untergeordnete Bedeutung besitzen.
\end{abstract}

\section{Einleitung}

Schwingungen, die sich durch feste Körper zum Aufenthaltsbereich von Menschen hin ausbreiten, können von diesen einerseits direkt als Erschütterungen und andererseits als von der Oberfläche einer Bauwerksstruktur (i. a. von einer Decke oder Wand) abgestrahlter sekundärer Luftschall wahrgenommen werden. Dabei werden die Erschütterungen, da es ein eigenständiges Erschütterungsorgan nicht gibt, taktil durch 
Tabelle 1. Menschliche Wahrnehmung und Empfindung von direktem Luftschall, Erschütterungen und Sekundärschall

\begin{tabular}{lll}
\hline Immissionsform & Wahrnehmung & Empfindung \\
\hline $\begin{array}{l}\text { direkter Luftschall } \\
\text { (Lärm) }\end{array}$ & auditiv durch das Ohr & $\begin{array}{l}\text { Fernreiz, Ortung, Flucht } \\
\text { möglich }\end{array}$ \\
\hline Erschütterung & $\begin{array}{l}\text { taktil durch den ganzen } \\
\text { Körper }\end{array}$ & $\begin{array}{l}\text { Nahreiz, Abwehrreaktion } \\
\text { unmöglich }\end{array}$ \\
\hline Sekundärschall & auditiv durch das Ohr & $\begin{array}{l}\text { Nahreiz, Abwehrreaktion } \\
\text { unmöglich }\end{array}$ \\
\hline
\end{tabular}

den ganzen Körper und der Sekundärschall - ebenso wie der direkte Luftschall - auditiv durch das Ohr aufgenommen. Diese beiden Wahrnehmungsformen bilden somit zwei Aspekte der gesamtheitlichen Erschütterungseinwirkung und somit eine kombinierte Exposition.

Über die Wahrnehmung hinaus ist auch die Empfindung des Menschen für diese Immissionsformen zu berücksichtigen. Dabei ergeben sich Empfindungsunterschiede bezüglich des direkten Luftschalls einerseits und der Erschütterungen sowie des Sekundärschalls andererseits.

Beim direkten Luftschall, der seiner großen Reichweite wegen als Fernreiz bezeichnet werden kann, sind im Freien die Ortung der Schallquelle und in Gebäuden Schutzmaßnahmen wie Fensterschließen möglich: Es wird dabei der Urinstinkt der Fluchtmöglichkeit angesprochen. Erschütterungen und der Sekundärschall stellen hingegen Nahreize dar, denen gegenüber man das Gefühl des Ausgeliefertseins erleben kann. Klassische Lärmschutzmaßnahmen wie Lärmschutzfenster sind zwecklos. Bei den Erschütterungen kommt noch hinzu, dass das biometrische Schwingungsverhalten einzelner Körperteile (Resonanzphänomene) Wahrnehmung und Empfindung unter Umständen beeinflussen können.

In Tab. 1 werden diese unterschiedlichen Wahrnehmungs- und Empfindungsformen schematisch dargestellt [1].

Dass es sich dabei um die Empfindungskategorien Lästigkeit und Störung handelt, kommt sprachlich bereits in den Bezeichnungen Lärm und Erschütterungen zum Ausdruck, die für positive Empfindungen wie „Musik hören“ oder ,,in den Schlaf wiegen“ nicht verwendet werden.

\section{Hörbarkeit und Fühlbarkeit}

Um diese Kombinationswirkung objektiv beurteilen zu können, ist es wie bei allen Immissionsformen zuerst erforderlich, sie quantitativ zu 
erfassen, d. h. operational durch Messverfahren, Zu- oder Abschläge und/oder Bewertungsfaktoren zu bestimmen. Dies wird durch die oben dargestellte unterschiedliche Form der Wahrnehmung erschwert, weshalb derzeit erst Aussagen zu Teilaspekten möglich sind. Die allgemeine Interaktion von Lärm und Erschütterungen wird für die Wahrnehmungsempfindung von verschiedenen Autoren unterschiedlich beantwortet, wie aus verschiedenen Übersichtsartikeln übereinstimmend hervorgeht [1-3].

Da die verschiedenen Untersuchungen naturgemäß unterschiedliche Teilaspekte betreffen, ergibt sich außerdem ein lückenhaftes Bild der Kombinationswirkung. Bezüglich der Erschütterungswahrnehmung ergibt sich durch Schallpegel $L_{A} \geq 64 \mathrm{~dB}$ nach MELONI [4] ein Verdeckungseffekt. STAHL et al. [5] untersuchen die Frage, ob ein Zusammenhang zwischen der Kombinationswahrnehmung von Schall und Erschütterungen, der daraus abgeleiteten Distanzwahrnehmung und der erlebten Lästigkeit besteht. Nach SCHUST et al. [6] ergibt sich bei der Kombinationswirkung ein zunehmender Einfluss der Tonhaltigkeit auf die Beurteilung von Lautheit und Belästigung.

Zumindest bei energetisch schwachen Immissionen (z. B. Verkehr) können in der Praxis belastete Personen oft nicht unterscheiden, ob sie etwas gehört oder am Körper gespürt haben. Die Begriffe „Hören“ und „Spüren“ werden von den Betroffenen oft unterscheidungslos verwendet. Dazu kommt, dass die Wahrnehmbarkeit von Erschütterungen sehr stark situationsabhängig ist. Nur bei gespannter Aufmerksamkeit wird eine Erschütterung der $W_{m}$-bewerteten Schwingbeschleunigung von $3,6 \mathrm{~mm} / \mathrm{s}^{2}$ nach ON-ISO 2631-1 tatsächlich wahrgenommen werden können. Jede Ablenkung (Zuhören, Lesen etc.) vermindert die Wahrnehmbarkeit bereits beträchtlich und eigene Aktivitäten (Reden etc.) noch mehr. Dementsprechend kann die Fühlschwelle nicht als Schwellwert, sondern nur als Schwellenband angegeben werden. Die obere Bandbegrenzung liegt etwa bei einer bewerteten Schwingbeschleunigung von $15 \mathrm{~mm} / \mathrm{s}^{2}$ [7], wie dies bei verschiedenen Untersuchungen immer wieder erkennbar ist [8].

\section{Schienenverkehrsimmissionen}

Hinsichtlich der Schienenverkehrsimmissionen liegt eine umfassende Studie von SAID et al. [9] vor, in deren Rahmen neben dem Auflösungsvermögen der menschlichen Wahrnehmung für die Stärke von Erschütterungen auch die Kombinationswirkung von Lärm und Erschütterungen auf den Menschen untersucht worden ist. Der Versuchsablauf wurde dabei nach den Methoden der Psychophysik so 
angelegt, dass neben der Sinnesempfindlichkeit der Probanden auch ihre Reaktionsbereitschaft erfasst werden konnte, da bei Diskriminationsaufgaben ,,entdeckungsfreudige“ Personen mögliche Differenzen schneller melden als ,zurückhaltende" Personen, die sich ihrer Sache zuerst sicher sein wollen.

Es wurde die Unterscheidung von insgesamt vier Erschütterungsintensitätsstufen $\left(K_{B, \max }=0,2 / 0,4 / 0,8 / 1,6\right.$ entsprechend $a_{W_{m, \text { max }}}=$ $7,1 \mathrm{~mm} / \mathrm{s}^{2}, 14 \mathrm{~mm} / \mathrm{s}^{2}, 29 \mathrm{~mm} / \mathrm{s}^{2}$ und $57 \mathrm{~mm} / \mathrm{s}^{2}$ bei drei $A$-bewerteten Vorbeifahrtspegeln $(30,45$ und $55 \mathrm{~dB})$ geprüft. Die Bandbreite der Stärke beider Immissionsformen umfasst somit einen Bereich, der im Umweltschutz häufig zu nachdrücklichen Klagen führt. Die Versuche ergaben

- A-bewertete Vorbeifahrtspegel von $30 \mathrm{~dB}$ sind ohne Einfluss auf die Unterscheidung der Erschütterungsintensität.

- A-bewertete Vorbeifahrtspegel von $45 \mathrm{~dB}$ beeinflussen die Unterscheidung ab einer $W_{m}$-bewerteten Schwingbeschleunigung von $29 \mathrm{~mm} / \mathrm{s}^{2}$ aufwärts.

- A-Bewertete Vorbeifahrtspegel von $55 \mathrm{~dB}$ reduzieren die Unterscheidungsfähigkeit um mehr als $20 \%$.

- Trotz verhältnismäßig hoher Intensitäten beider Immissionsformen sind die Kombinationseffekte eher tendenziell als signifikant nachweisbar gewesen.

In einer Untersuchung über die Lärm-Erschütterungs-Kombinationswirkung von Shinkansen-Hochgeschwindigkeitszügen von YOKOSHIMA und TAMURA [10] wird ebenfalls berichtet, dass die Shinkansen-Züge negativer zu bewerten sind als der sonstige Schienenverkehr, wo die Immissionen geringere Stärke besitzen. Da in dieser Studie einerseits unbewertete Schwingbeschleunigungsdaten für die Erschütterungsimmissionen verwendet wurden und andererseits die zugrunde liegende Bausubstanz der japanischen Studie (87\% Holzhäuser) für europäische Verhältnisse untypisch ist, sind die sonstigen Ergebnisse der Untersuchung nicht mit den europäischen Arbeiten vergleichbar.

Eine weitere Untersuchung zur Kombinationswirkung von Schall und Erschütterungen des Schienenverkehrs haben HOWARTH und GRIFFIN [11] mit dem Ziel durchgeführt festzustellen, welche der beiden Immissionsformen stärker belästigend wirkt und deshalb vordringlich zu bekämpfen ist. Es wurden Zugsvorbeifahrten von 24 Sekunden Dauer mit jeweils 6 verschiedenen Vorbeifahrtspegeln des Schalls bzw. 6 unterschiedlichen Erschütterungsintensitäten hinsichtlich der Belastigungseinstufung durch die Probenden untersucht. Die 


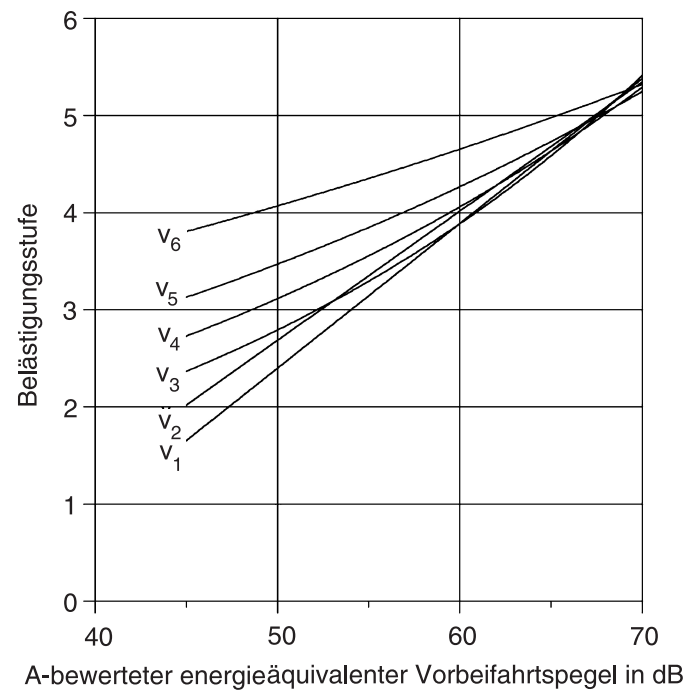

Abb. 1. Einfluss der Erschütterungsintensität auf die Gesamtbelästigung kombinierter Schall- und Erschütterungsimmissionen für $W_{m}$-bewertete Schwingbeschleunigungen (nach [11]) $\left(v_{1}: 23 \mathrm{~mm} / \mathrm{s}^{2} ; v_{2}: 32 \mathrm{~mm} / \mathrm{s}^{2} ; v_{3}: 45 \mathrm{~mm} / \mathrm{s}^{2} ; v_{4}: 65 \mathrm{~mm} / \mathrm{s}^{2}\right.$; $v_{5}: 90 \mathrm{~mm} / \mathrm{s}^{2} ; v_{6}: 130 \mathrm{~mm} / \mathrm{s}^{2}$

untersuchte Bandbreite sowohl der Erschütterungsintensität als auch des Schallpegels liegt oberhalb jener der Studie von SAID. Da dabei die Erschütterungsintensität in der gemäß ON-ISO 2631-1 nicht anzuwendenden Kenngröße VDV (Vibration Dose Value) angegeben ist, muss diese zur besseren Vergleichbarkeit in die $W_{m}$-bewertete Schwingbeschleunigung transformiert werden. Dies kann, da die beiden Einheiten in keinem formelmäßigen Zusammenhang stehen, nur näherungsweise nach einer Schätzformel [3] erfolgen. Das Ergebnis ist in den Abb. 1 und 2 wiedergegeben.

Nach Abb. 1 ergibt sich für $A$-bewertete Vorbeifahrtspegel von $45 \mathrm{~dB}$ eine deutliche Erhöhung der Belästigung durch gleichzeitige Erschütterungsimmissionen von $65 \mathrm{~mm} / \mathrm{s}^{2}$ aufwärts. Diese zusätzliche Belästigung nimmt mit anwachsendem Schallpegel $a b$, und spätestens $a b$ $65 \mathrm{~dB}$ beeinflussen die Erschütterungsimmissionen den Grad der Belästigung nicht mehr (Verdeckungseffekt). Bei niedrigen Erschütterungsintensitäten bis etwa $45 \mathrm{~mm} / \mathrm{s}^{2}$ ist kein statistisch signifikanter Einfluss auf die Belästigung durch die Schallimmissionen erkennbar, wie die sich mehrfach schneidenden Regressionskurven erkennen lassen.

Analog ergibt sich nach Abb. 2 für die $W_{m}$-bewertete Schwingbeschleunigung bei etwa $25 \mathrm{~mm} / \mathrm{s}^{2}$ eine deutliche Erhöhung der Belästi- 


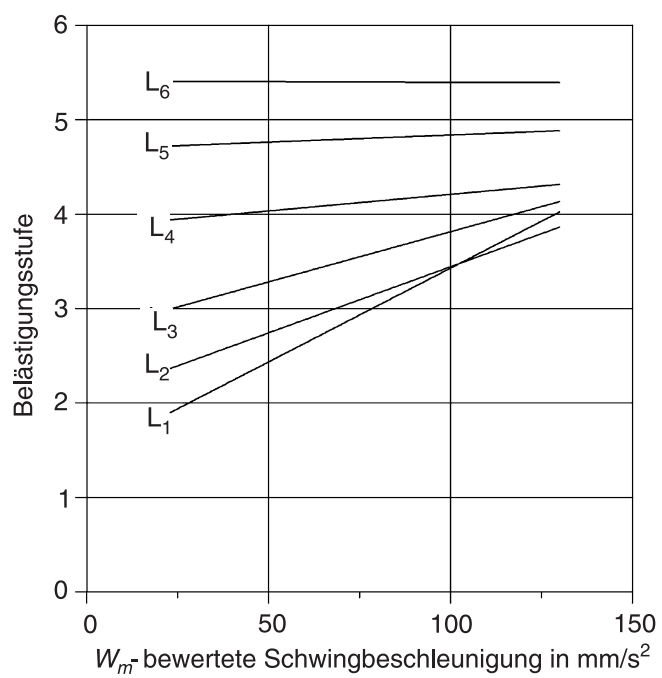

Abb. 2. Einfluss des Schallpegels auf die Gesamtbelästigung kombinierter Schallund Erschütterungsimmissionen für A-bewertete Vorbeifahrtspegel (nach [11]) $\left(L_{1}: 45 \mathrm{~dB} ; L_{2}: 50 \mathrm{~dB} ; L_{3}: 55 \mathrm{~dB} ; L_{4}: 60 \mathrm{~dB} ; L_{5}: 65 \mathrm{~dB} ; L_{6}: 70 \mathrm{~dB}\right)$

gung durch gleichzeitige Schallimmissionen. Auch hier verringert sich die zusätzliche Belästigung mit anwachsender Erschütterungsintensität, wobei die Erschütterungen ab einem $A$-bewerteten Schallpegel von etwa $60-65 \mathrm{~dB}$ für Belästigung insignifikant werden und somit den in Abb. 1 erkennbaren Verdeckungseffekt bestätigen. Auch im Fall der Erschütterungsimmissionen ergeben niedrige Vorbeifahrtsschallpegel (45-50 dB) keinen signifikanten Einfluss auf die Belästigung, wie die sich schneidenden, eng beeinander liegenden Regressionskurven belegen.

Aus diesen Untersuchungen zusammen ergibt sich somit ein komplexes Bild der Kombinationswirkung. Im Bereich von Erschütterungsintensitäten unterhalb des Schwellenbands der Fühlbarkeit kann es definitionsgemäß keine Kombinationswirkung geben. Erschütterungen geringer, jedoch spürbarer Intensität können von Schallimmissionen praktisch nicht unterschieden werden, sodass keine Wechselwirkung beobachtet werden kann [1]. Erst bei mittleren Erschütterungsintensitäten (ca. $a_{W_{m}}=30 \mathrm{~mm} / \mathrm{s}^{2}$ ) bzw. Schallpegeln (ca. $L_{A_{\mathrm{eq}}}=45 \mathrm{~dB}$ ) fächert die Belästigungswirkung in Abhängigkeit von beiden Immissionsgrößen auf, bis ab $A$-bewerteten Schallpegeln von etwa 60-65 dB ein Verdeckungseffekt des Schalls wirksam wird.

Das interessante Ergebnis dieser Untersuchung ist es, dass die Versuchspersonen der Erschütterungsreduktion bei eher schwachen 
Erschütterungsimmissionen und der Lärmreduktion bei hohen Lärmimmissionen den Vorzug geben.

\section{Schlussfolgerungen}

Hinsichtlich der Kombinationswirkung von Erschütterungen und Sekundärschall können die Ergebnisse der hier diskutierten Untersuchungen folgendermaßen zusammengefasst werden.

1. Bei schwachen Immissionen (z. B. Verkehr) kann der Mensch zwischen hörbaren Schall- und fühlbaren Erschütterungsimmissionen nicht unterscheiden, weshalb eine spezielle Kombinationswirkung nicht fassbar ist.

2. Eine Wechselwirkung zwischen Erschütterungs- und Schallimmissionen ist erst ab $W_{m}$-bewerteten Schwingbeschleunigungen von $30 \mathrm{~mm} / \mathrm{s}^{2}$ aufwärts zu erwarten, wenn die $A$-bewerteten Vorbeifahrtsschallpegel auf $45 \mathrm{~dB}$ und darüber anwachsen.

3. Wenn eine der beiden Immissionsgrößen auf niedrigem Niveau bleibt, wie dies beim Sekundärschall praktisch immer zutrifft, dann ergibt sich keine signifikante Kombinationswirkung.

4. Die Immissionsschutzkriterien von ÖNORM S 9012 für Erschütterungs- und Sekundärschallimmissionen des Schienenverkehrs liegen unter den oben angegebenen Werten. Es ist daher nicht erforderlich, eine Kombinationswirkung beider Immissionsformen zusätzlich zu berücksichtigen.

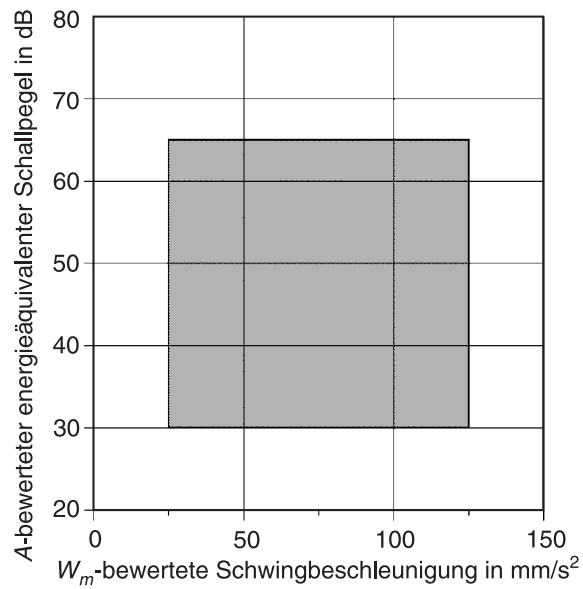

Abb. 3. Bereich der $W_{m}$-bewerteten Schwingbeschleunigung und der $A$-bewerteten Schallpegel, für den weitere qualitative und quantitative Untersuchungen der Kombinationswirkung erforderlich sind 
134 P. Steinhauser: Zur Kombinationswirkung von Schall und Erschütterungen

5. Hohe Schallpegel (ab etwa 60-65dB) bewirken einen Verdeckungseffekt für Erschütterungen.

6. Die Quantifizierung der Kombinationswirkung innerhalb des in Abb. 3 angegebenen Immissionsbereichs ist noch durchzuführen.

Insgesamt gilt immer noch die Feststellung von GRIFFIN [11], die sinngemäß lautet: „Die potenziellen Wechselwirkungen der Reaktion auf Lärm und Erschütterungen sind komplex. Der Nachweis solcher Effekte ist schwach und teilweise widersprüchlich. " Beispielsweise sind weitere Untersuchungen zur Kombinationswirkung für niedrige und mittlere Immissionsstärken (siehe Abb. 3) von Schall und Erschütterungen erforderlich.

\section{Literatur}

[1] MelonI, T. (2006) Bericht der Fachgruppe zur Verordnung über den Schutz vor Erschütterungen. Bundesamt für Umwelt BAFU, Abt. Lärmbekämpfung, Bern

[2] Haider, M., Koller, M., STIDL, H. G. (1992) Qualitätskriterien für Schienenverkehrslärm und Erschütterungen bei Vollbahnen - Kombinationswirkungen von Lärm und Erschütterungen. Forschungsarbeiten aus dem Verkehrswesen, Band 36/1, Wien

[3] GRIFFIN, M. J. (1990) Handbook of Human Vibrations. Academic Press, San Diego

[4] MelonI, T. (1991) Wahrnehmung und Empfindung von komplexen, kombinierten Belastungen durch Vibrationen und Schall. Dissertation, ETH Zürich

[5] Stahl, E., Meloni, T., Krueger, H. (1997) Distanzwahrnehmung von Umweltsituationen. Zs. f. Lärmbekämpfung 44/1

[6] SCHUST, M., SEIDEL, H., BLÜTHNER, R. (1998) Wirkung von Lärm unterschiedlicher Tonhaltigkeit nach DIN 45681 in Kombination mit Schwingungen. Zs. f. Lärmbekämpfung 45/4

[7] ON-ISO 2631-1 (2007) Mechanische Schwingungen und Stöße - Bewertung der Auswirkung von Ganzkörperschwingungen auf den Menschen, Teil 1, Anhang $\mathrm{C}$

[8] Berger, P., LANG, J., ÖSterReicher, M., Steinhauser, P. (2005) Wirksamkeit der Schutzmaßnahmen gegen U-Bahn-Immissionen für den Wiener Musikverein. Zement und Beton 2/05

[9] SAID, A., Fleischer, D., KILCHER, H., GRÜTZ, H. P. (2001) Zur Bewertung von Erschütterungsimmissionen aus dem Schienenverkehr. Zs. f. Lärmbekämpfung $48 / 6$

[10] Yokoshima, S., Tamura, A. (2005) Combined annoyance due to the Shinkansen railway noise and vibration. Internoise Congress, Rio de Janeiro.

[11] HowarTh, H. V. C., GRIFFIN, M. J. (1990) The relative importance of noise and vibration from railways. Applied Ergonomics 21/2

Anschrift des Verfassers: Univ.-Prof. Dr. Peter Steinhauser, Delugstraße 8, 1190 Wien, Österreich. E-Mail: Peter.Steinhauser@univie.ac.at. 